\title{
Therapeutic potentials of n-hexane extracts of the three medicinal mushrooms regarding their anti-colon cancer, antioxidant, and hypocholesterolemic capabilities
}

\author{
GHOSON M. DABA ${ }^{1}$, WAILL A. ELKHATEEB ${ }^{1, \bullet}$, ASMAA NEGM EL-DEIN ${ }^{1}$, EMAN F. AHMED ${ }^{1}$, \\ ALI MOHAMED EL HAGRASSI ${ }^{2}$, WALID FAYAD ${ }^{3}$, TING-CHI WEN, ${ }^{4, \bullet}$ \\ ${ }^{1}$ Chemistry of Natural and Microbial Products Department, Pharmaceutical Industries Division, National Research Centre. Dokki, Giza, 12622, Egypt. \\ •email: waillahmed@yahoo.com \\ ${ }^{2}$ Phytochemistry and Plant Systematic Department, Pharmaceutical industries Division, National Research Centre. Dokki, Giza, 12622, Egypt. \\ ${ }^{3}$ Drug Bioassay-Cell Culture Laboratory, Pharmacognosy Department, National Research Centre. Dokki, Giza, 12622, Egypt. \\ ${ }^{4}$ Engineering Research Center of Southwest Bio-Pharmaceutical Resources, Guizhou University. Guiyang 550025, Guizhou Province, China. \\ vemail: tingchiwen@yahoo.com
}

Manuscript received: 29 April 2020. Revision accepted: 11 May 2020.

\begin{abstract}
Daba GM, Elkhateeb WA, El-Dien AN, Ahmed EF, El Hagrassi AM Fayad W, Wen TC. 2020. Therapeutic potentials of nhexane extracts of the three medicinal mushrooms regarding their anti-colon cancer, antioxidant, and hypocholesterolemic capabilities. Biodiversitas 21: 2437-2445. Evaluating in vitro biological activities of the medicinal mushrooms Hericium erinaceus, Metacordyceps neogunnii, and Dictyophora indusiata $\mathrm{n}$-hexane extracts revealed their capabilities as promising therapeutic sources. By assessing their DPPH radical scavenging activities, $D$. indusiata extract exhibited the highest antioxidant activity $(87.8 \pm 1.2 \%)$ followed by $H$. erinaceus, then M. neogunnii $84.9 \pm 1.6 \%$, and $77.3 \pm 1.3 \%$, respectively. On the other hand, M. neogunnii extract exerted promising anticolon cancer $(68.6 \pm 3.6 \%$ cytotoxicity) against HCT116 human colon cancer cell lines at concentration $100 \mu \mathrm{g} / \mathrm{mL}$, whereas $H$. erinaceus and $D$. indusiata extracts exhibited weaker cytotoxic effects $(18.3 \pm 1.7 \%$, and $19.3 \pm 3.2 \%$, respectively) using the same concentration. Investigating cholesterol reduction activities (CRA\%) of the extracts revealed that activity depends on both extract concentration and incubation period. After $96 \mathrm{~h}$ of incubation at room temperature, all extracts exerted potent in vitro hypocholesterolemic activities (100 $\pm 0 \%$ reduction in cholesterol concentration). Performing GC-Ms analyses on the extracts revealed presence of 22 compounds in D. indusiata extract, while 29 compounds were detected in M. neogunnii extract, and 33 in $H$. erinaceus extract. Most of compounds were esters of fatty acids. Results in this study encourage using these mushrooms as functional foods, and highlight the need for conducting further in vivo studies to support their use in pharmaceutical industry.
\end{abstract}

Keywords: Anti-colon cancer; antioxidant; Dictyophora indusiata; Hericium erinaceus, hypocholesterolemic; Metacordyceps neogunnii

\section{INTRODUCTION}

Reducing cholesterol levels is one of the key factors controlling cardiovascular diseases (CVD) through decreasing risk of developing some heart diseases (Jeong et al. 2018). Reports of WHO have specified CVD as the reason for mortalities of about 18 million people annually which represents $31 \%$ of total number of mortalities all over the world (WHO fact sheet, 2017). Currently used HMG-CoA reductase inhibitors (statins) prescribed for cholesterol-lowering purpose have some serious side effects such as statin-associated muscle symptoms, pancreatic and hepatic dysfunction, diabetes mellitus, neuropathy, sexual dysfunction, cognitive loss, and central nervous system complaints (Golomb and Evans, 2008; Thompson et al. 2016). These serious adverse effects made researchers investigate for different agents having cholesterol-reducing capabilities. On the other hand, colon cancer is one of the major cancers responsible for elevated number of mortalities worldwide. Colorectal cancer alone is the cause of mortalities approaching 1 million people in 2018 (WHO fact sheet, 2018). Hence, all efforts are focusing nowadays on screening for sources rich in compounds displaying anticancer properties.

Mushrooms are group of macrofungi belong to divisions Ascomycota and Basidiomycota. Consumption of mushrooms as food or for their medicinal properties is known especially in Asian countries from millennia (Chang and Miles, 1989; Sullivan et al. 2006; Wang et al. 2018; Elkhateeb et al. 2019a). Metacordyceps neogunnii, or as known previously, Paecilomyces gunnii (its asexual morph, Liang 1985), is an entomogenous fungus used in Asian countries as a medicinal mushroom (Wen et al. 2017). For over 30 years, this mushroom has been wrongly regarded as Cordyceps gunnii but currently, it has been proven that it differs morphologically from similar species since it has longer asci and wider ascospores. Furthermore, multigene analysis and sequence data had confirmed the uniqueness of this species (Wen et al. 2017). Generally, Chinese Cordyceps species are ranked among the expensive natural alternatives due to their medicinal capabilities (Bashir et al. 2017). One kilogram of this mushroom costs about $2000 \$$, and it is recognized as an alternative for the famous mushroom Ophiocordyceps sinensis. However, precise studies discussing the biological activities of 
Metacordyceps neogunnii extracts are remarkably rare. One of the important edible mushrooms is Hericium erinaceus which is a well-studied medicinal mushroom known for centuries in Chinese traditional medicine as well as South American and European literature (Thongbai et al. 2015). Many biological activities have been reported for the fruiting bodies extracts of $H$. erinaceus such as having haemagglutinating, anti-aging, immunomodulatory, hypoglycemic, antimicrobial, antiproliferative, and antitumor activities (Mizuno et al. 1992; Gong et al. 2004; Yim et al. 2007; Li et al. 2010; Zhang et al. 2012; Thongbai et al. 2015; Elkhateeb et al. 2019b).

Another fascinating mushroom is Dictyophora indusiata which is famous for its medical importance since 618 AD (Han et al. 2017). The extract of D. indusiata has been reported to have antimicrobial, and antioxidant activities (Oyetayo et al. 2009; Elkhateeb et al. 2020). Moreover, polysaccharides extracted from $D$. indusiata have great potentiality to be applied in functional food.

In this study, metabolites profiles of n-hexane extracts of the three mushrooms Metacordyceps neogunnii, Hericium erinaceus, and Dictyophora indusiata were elucidated using gas chromatography-mass spectrometry (GC-MS) analysis. At the same time, in vitro cholesterollowering capabilities, antioxidant activities, and antihuman colon cancer abilities of their n-hexane extracts were investigated.

\section{MATERIALS AND METHODS}

\section{Study area}

This study was conducted in the laboratories of the national research center of Egypt. In the departments of chemistry of natural and microbial products, phytochemistry and Plant Systematic, Drug Bioassay-Cell Culture Laboratory, pharmacognosy Department. Also, in the laboratory of engineering Research Center of Southwest Bio-Pharmaceutical Resources, Guizhou University in China.

\section{Sample and extraction}

Metacordyceps neogunnii, Hericium erinaceus and Dictyophora indusiata were kindly supplied by Prof. Dr. Ting-Chi Wen from Guizhou, Tianqi, Wildlife, Sources, Conservation R\&D Centre in Guiyang city, Guizhou Province, China (Figure 1).

Two hundred and fifty grams of each mushroom fruiting bodies were washed with distilled water, air-dried before cut into small pieces, and placed in an Erlenmeyer flask containing n-hexane at room temperature, and kept for $48 \mathrm{~h}$ prior to filtering. The resulting filtered extracts were concentrated at $37{ }^{\circ} \mathrm{C}$ using a rotary evaporator. Obtained extracts were stored at $4^{\circ} \mathrm{C}$ in clean closed containers until further use.

\section{GC-MS analysis}

The GC-MS analyses of the n-hexane extracts were performed using a gas chromatography-mass spectrometry instrument in the Department of Medicinal and Aromatic

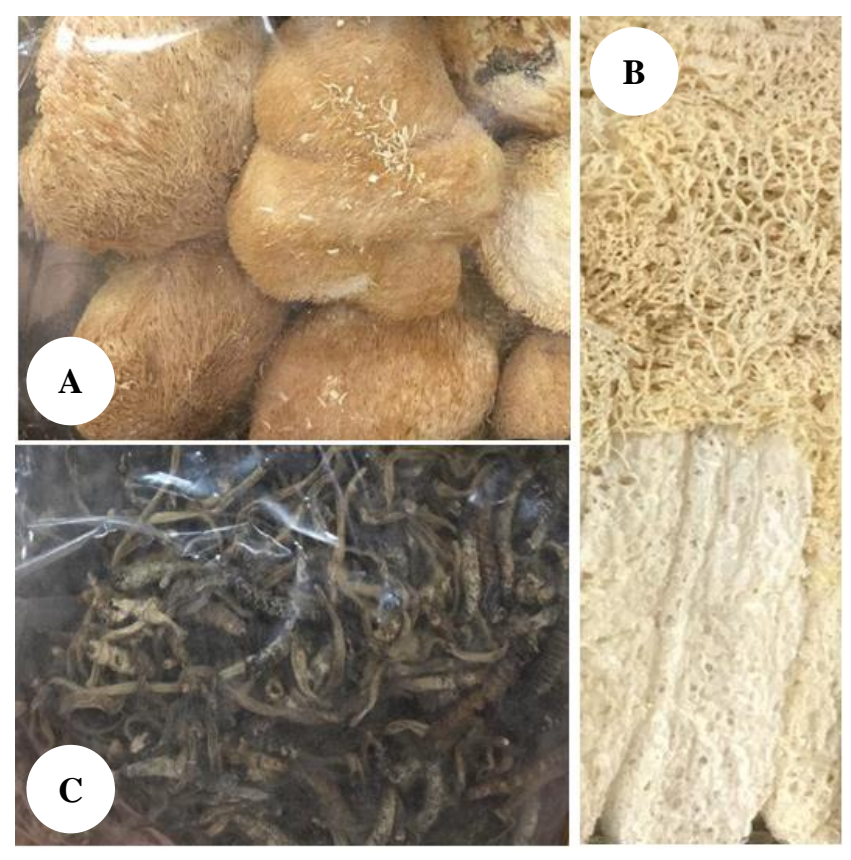

Figure 1. Medicinal mushrooms used in this study: A. Hericium erinaceus, B. Dictyophora indusiata, and C. Metacordyceps neogunnii. Photographs were taken by WAE

Plants Research, National Research Center, Egypt with the following specifications. Instrument: a TRACE GC Ultra Gas Chromatographs (THERMO Scientific Corp., USA), coupled with a THERMO mass spectrometer detector (ISQ Single Quadrupole Mass Spectrometer). The GC-MS system was equipped with a TG-WAX MS column (30 m x $0.25 \mathrm{~mm}$ i.d., $0.25 \mu \mathrm{m}$ film thickness). Analyses were carried out using helium as carrier gas at a flow rate of 1.0 $\mathrm{mL} / \mathrm{min}$ and a split ratio of 1:10 using the following temperature program: $60{ }^{\circ} \mathrm{C}$ for $1 \mathrm{~min}$; rising at $3.0^{\circ} \mathrm{C} / \mathrm{min}$ to $240^{\circ} \mathrm{C}$ and held for $1 \mathrm{~min}$. The injector and detector were held at $240{ }^{\circ} \mathrm{C}$. Diluted samples (1:10 hexane, v/v) of $0.2 \mu \mathrm{L}$ of the mixtures were always injected automatically in splitless mode. Mass spectra were obtained by electron ionization (EI) at $70 \mathrm{eV}$, using a spectral range of $\mathrm{m} / \mathrm{z} 40$ 450. Most of the compounds were identified using the analytical method: mass spectra (authentic chemicals, Wiley spectral library collection, and NSIT library). The quantification of the components was based on the metabolites as detected by the mass spectrometer. Identification of the constituents was carried out by comparison of their retention times and fragmentation pattern of mass with those of published data assay (Adams, 1995) and or with those of the Wiley 9 and NIST08 mass spectra library.

\section{Antioxidant scavenging activity of mushrooms n-hexane extracts}

DPPH (1-diphenyl-2-picrylhydrazyl) scavenging activity was measured as described previously by Lee et al. (2010). Briefly, $500 \mu \mathrm{l}$ of ethanolic DPPH solution $(0.4$ mmol) was mixed vigorously with $500 \mu$ l of each mushroom extract $(80 \mathrm{mg} / \mathrm{mL})$ or water (as a control) and 
incubated at $37^{\circ} \mathrm{C}$ in the dark for $1 \mathrm{~h}$. The absorbance of the mixture was measured spectrophotometrically at $517 \mathrm{~nm}$. The scavenging activity was calculated as; Scavenging activity $(\%)=\left[1-\left(\mathrm{A}_{\mathrm{s}}-\mathrm{A}_{\mathrm{b}}\right) / \mathrm{A}_{\mathrm{c}}\right] \times 100$, whereas $\mathrm{A}_{\mathrm{b}}, \mathrm{A}_{\mathrm{c}}$ and $\mathrm{A}_{\mathrm{s}}$ is the absorbance of the blank (ethanol and sample), the control (DPPH and deionized water) and the sample (DPPH and sample), respectively. Ascorbic acid at the concentration of $0.1 \%$ was used as positive control.

\section{In vitro anti-humann colon cancer activity of mushrooms n-hexane extracts Cell culture}

HCT116 human colon carcinoma cell lines were cultured in $95 \%$ humidity, $5 \% \mathrm{CO}_{2}$ and $37^{\circ} \mathrm{C}$. The cell line was maintained in McCoy's 5A medium supplemented with $10 \%$ fetal bovine serum.

\section{Cytotoxicity on HCT116 cell line monolayers}

The acid phosphatase assay was used to assess cytotoxicity according to the method described by Yang et al (2012). A number of cells (1000) were seeded per well in 96 well plates, left to attach overnight, and then treated with mushrooms extracts for three days. For one plate, a substrate solution was prepared, where $20 \mathrm{mg}$ tablet of pNPP (Sigma; cat. no. N2765) was dissolved in $10 \mathrm{~mL}$ buffer solution $(0.1 \mathrm{M}$ sodium acetate, $0.1 \%$ Triton $\mathrm{X}-100$, $\mathrm{pH}=5)$. Cell monolayers were washed with $250 \mu \mathrm{l}$ PBS. $100 \mu \mathrm{l}$ of pNPP substrate solution was added per well, then plates were incubated for 4 hours at $37^{\circ} \mathrm{C}$. Sodium hydroxide $(1 \mathrm{~N})$ stop solution was used by adding $10 \mu \mathrm{l}$ per well. Absorbance was measured directly at wavelength 405 $\mathrm{nm}$. All samples were tested in triplicates, and 0.5\% DMSO was used as negative control, and $50 \mu \mathrm{M}$ cisplatin was used as positive control. Samples were tested at serial dilutions with final concentration of $100,50,25$, and $12.5 \mu \mathrm{g} / \mathrm{mL}$.

Percent cytotoxicity was calculated by the formula:

$$
[1-(\mathrm{D} / \mathrm{S})] \times 100
$$

Where, D and S denote the optical density of drug and solvent treated wells, respectively.

\section{In vitro hypocholesterolemic abilities of mushrooms n- hexane extracts.}

Different concentrations of each mushroom extract $(0.5-4.0 \%)$ were prepared as described previously (Elkhateeb et al. 2019c). Soluble cholesterol (1 mL) was added to $4 \mathrm{~mL}$ of each concentration and incubated at room temperature for $24,48,72$, and $96 \mathrm{hrs}$. To determine the residual amount of cholesterol, the cholesterol assay kit (Biodiagnostic, Egypt) was used and the percentage of cholesterol-reducing activity was assessed. The control was prepared by adding $4 \mathrm{~mL}$ distilled water to $1 \mathrm{~mL}$ soluble cholesterol. The percentage of cholesterol-reducing activity was calculated as describes previously (Pan and Zhang 2005) as follows:

Cholesterol reducing activity $(\%)=\left[\left(\mathrm{A}_{0}-\mathrm{A}\right) / \mathrm{A}_{0}\right] \times 100$

Whereas $\mathrm{A}_{0}$ : absorbance of the control $(500 \mathrm{~nm})$; A: absorbance of the sample $(500 \mathrm{~nm})$. Tests were carried out in triplicates.

\section{RESULTS AND DISCUSSION}

\section{GC-Ms analysis of the n-hexane extracts of $M$. neogunnii, $D$. indusiata and $H$. erinaceus}

Gc-Ms analyses were performed on the n-hexane extracts of the three mushrooms. As shown in Figure 2, and Table 1, 29 compounds were detected in the n-hexane extract of Metacordyceps neogunnii. The total peak areas of the detected compounds were $93.66 \%$, the probabilities of the structures of the detected compounds are listed in Table 1. The major peak areas are $11.57 \%$ Hexadecanoic acid, methyl ester $\mathrm{C}_{17} \mathrm{H}_{34} \mathrm{O}_{2}, \quad 15.94 \%$ 9,12-Octadecadienoic acid, methyl ester $\mathrm{C}_{19} \mathrm{H}_{34} \mathrm{O}_{2}, 50.74$ $\%$ 9-Octadecenoic acid (Z)-, methyl ester (Oleic acid, methyl ester) $\mathrm{C}_{19} \mathrm{H}_{36} \mathrm{O}_{2}, 3.05 \%$ 9,12-Octadecadienoic acid $(\mathrm{Z}, \mathrm{Z})$ (Linoleic acid $\left(\mathrm{C}_{18} \mathrm{H}_{32} \mathrm{O}_{2}\right.$, for which represented $81.48 \%$ of the total peak. On the other hand, GC-Ms analysis of the n-hexane extract of Hericium erinaceus (Figure 3, Table 2) resulted in detection of 33 compounds. The total peak areas of the detected compounds were 90.11 $\%$, the probabilities of the structures of the detected compounds are Pentane, 1-nitro- $\mathrm{C}_{5} \mathrm{H}_{11} \mathrm{NO}_{2} 18.27 \%$ and 1,6-Octadiene, 3,7-dimethyl- $\mathrm{C}_{10} \mathrm{H}_{18}, 14.23 \%$, and the major peak areas are for which represented $32.50 \%$ of the total peak areas (Figure 3). Finally, as shown in Figure (4), and Table 3, GC-Ms analysis of the n-hexane extract of Dictyophora indusiata resulted in identification of 22 compounds. The total peak areas of the detected compounds are $99.89 \%$, the probabilities of the structures of the detected compounds are listed in Table 3. The major peak areas are $82.85 \%$ for Butane, 1-isocyano- $\mathrm{C}_{5} \mathrm{H}_{9} \mathrm{~N}$, and $10.87 \%$ for Pentane, 1-nitro $\mathrm{C}_{5} \mathrm{H}_{11} \mathrm{NO}_{2}$ for which represented $93.72 \%$ of the total peak areas.

\section{Antioxidant scavenging activity of mushrooms n-hexane extracts}

The in vitro hydrogen-donating or radical scavenging activity of the three mushrooms n-hexane extracts was evaluated using the stable DPPH reagent. As shown in Figure (5), all tested mushrooms extract exerted very good antioxidant capabilities. D. indusiata exhibited a relatively higher antioxidant activity $(87.8 \pm 1.2 \%)$ at concentration 80 $\mathrm{mg} / \mathrm{mL}$, followed by $H$. erinaceus, then $M$. neogunnii which have recorded $84.9 \pm 1.6 \%$, and $77.3 \pm 1.3 \%$, respectively at the same concentration.

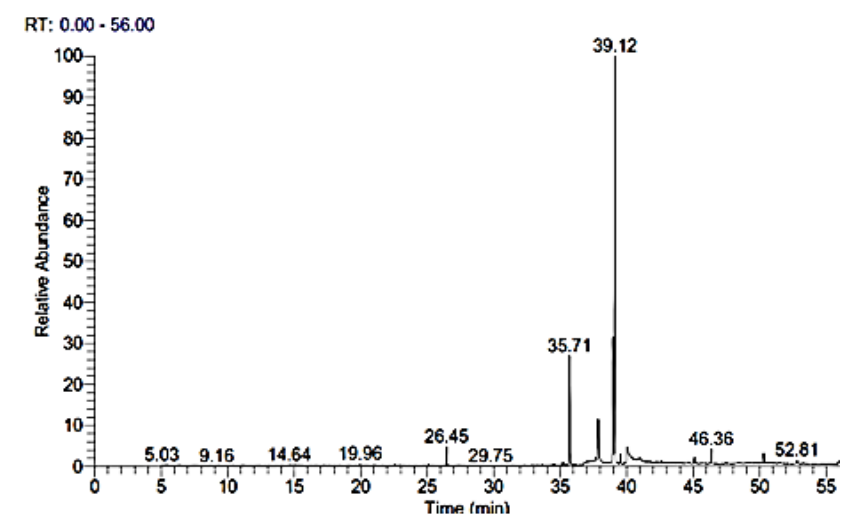

Figure 2. Total ion chromatogram of n-hexane extract of Metacordyceps neogunnii 


\section{In vitro anti-humann colon cancer activity of mushrooms n-hexane extracts}

The cytotoxic activities of the tested mushrooms nhexane extracts were assessed against HCT116 human colon carcinoma cell line. As shown in Fig (6), the anticancer activity was concentration-dependent. Using $M$. neogunnii extract at concentration of $100 \mu \mathrm{g} / \mathrm{mL}$ resulted in a cytotoxicity of $68.6 \pm 3.6 \%$ whereas both $D$. indusiata and $H$. erinaceus exhibited weaker cytotoxicity effects using the same concentration $(100 \mu \mathrm{g} / \mathrm{mL})$. Cytotoxicity caused by $D$. indusiata extract was $19.3 \pm 3.2 \%$ while that resulted from treatment with $H$. erinaceus extract was $18.3 \pm 1.7 \%$.

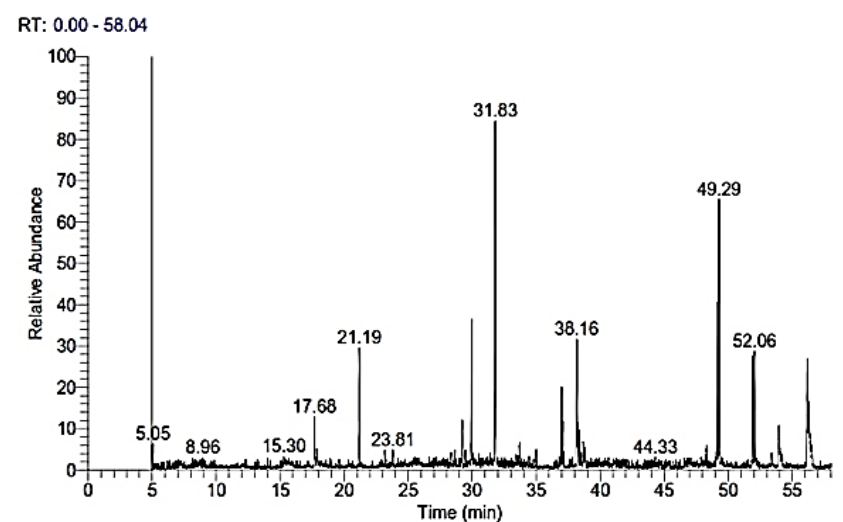

Figure 3. Total ion chromatogram of n-hexane extract of Hericium erinaceus

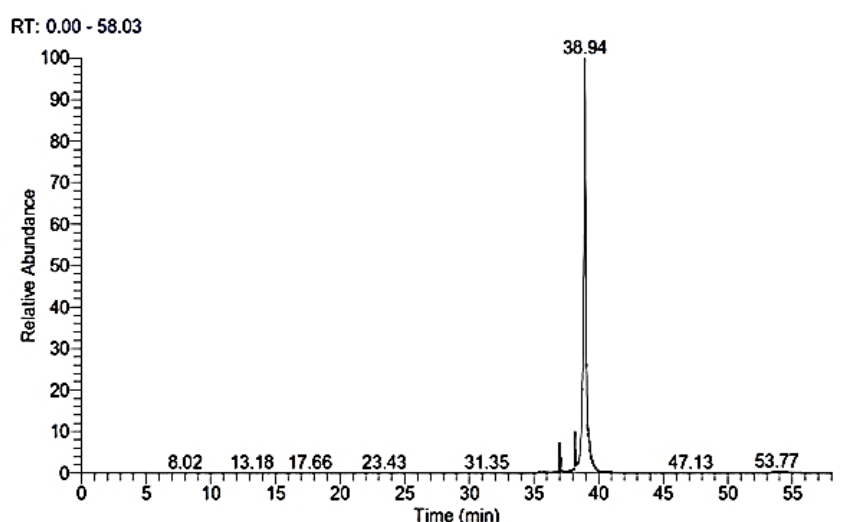

Figure 4. Total ion chromatogram of n-hexane extract of Dictyophora indusiata

Table 1. GC/MS analysis of Metacordyceps neogunnii n-hexane extract

\begin{tabular}{|c|c|c|c|c|c|}
\hline $\begin{array}{l}\text { Peak } \\
\text { No. }\end{array}$ & $\begin{array}{l}\text { Rt } \\
(\text { min. })\end{array}$ & MW & $\begin{array}{l}\text { Area } \\
\%\end{array}$ & $\begin{array}{l}\text { Molecular } \\
\text { formula }\end{array}$ & Probabilities of the detected compounds \\
\hline 1 & 26.45 & 204 & 2.01 & $\mathrm{C}_{14} \mathrm{H}_{20} \mathrm{O}$ & (3E)-5-Isopropyliden-6-methyl-3,6,9-decatrien-2-one \\
\hline 2 & 33.56 & 256 & 0.12 & $\mathrm{C}_{16} \mathrm{H}_{32} \mathrm{O}_{2}$ & Tetradecanoic acid, 12-methyl-, methyl ester \\
\hline 3 & 34.55 & 278 & 0.26 & $\mathrm{C}_{16} \mathrm{H}_{22} \mathrm{O}_{4}$ & 1,2-Benzenedicarboxylic acid, dibutyl ester \\
\hline 4 & 35.15 & 268 & 0.45 & $\mathrm{C}_{17} \mathrm{H}_{32} \mathrm{O}_{2}$ & 9-Hexadecenoic acid, methyl ester, (Z)- (Methyl palmitoleate) \\
\hline 5 & 35.71 & 270 & 11.75 & $\mathrm{C}_{17} \mathrm{H}_{34} \mathrm{O}_{2}$ & Hexadecanoic acid, methyl ester \\
\hline 6 & 36.21 & 468 & 0.10 & $\mathrm{C}_{32} \mathrm{H}_{52} \mathrm{O}_{2}$ & Lup-20 (29)-en-3-ol, acetate, (3á) \\
\hline 7 & 36.93 & 490 & 0.47 & $\mathrm{C}_{35} \mathrm{H}_{70}$ & 17-Pentatriacontene \\
\hline 8 & 37.03 & 310 & 0.40 & $\mathrm{C}_{22} \mathrm{H}_{46}$ & Docosane \\
\hline 9 & 37.17 & 280 & 0.20 & $\mathrm{C}_{18} \mathrm{H}_{32} \mathrm{O}_{2}$ & Methyl 9,12-heptadecadienoate \\
\hline 10 & 37.36 & 282 & 0.13 & $\mathrm{C}_{18} \mathrm{H}_{34} \mathrm{O}_{2}$ & cis-10-Heptadecenoic acid, methyl ester \\
\hline 11 & 37.64 & 284 & 0.42 & $\mathrm{C}_{18} \mathrm{H}_{36} \mathrm{O}_{2}$ & Heptadecanoic acid, methyl ester \\
\hline 12 & 38.96 & 294 & 15.94 & $\mathrm{C}_{19} \mathrm{H}_{34} \mathrm{O}_{2}$ & 9,12-Octadecadienoic acid, methyl ester (Linolelaidic acid, methyl ester) \\
\hline 13 & 39.12 & 296 & 50.74 & $\mathrm{C}_{19} \mathrm{H}_{36} \mathrm{O}_{2}$ & 9-Octadecenoic acid (Z)-, methyl ester (Oleic acid, methyl ester) \\
\hline 14 & 39.51 & 298 & 1.03 & $\mathrm{C}_{19} \mathrm{H}_{38} \mathrm{O}_{2}$ & Octadecanoic acid, methyl ester (Methyl stearate) \\
\hline 15 & 39.82 & 328 & 0.11 & $\mathrm{C}_{26} \mathrm{H}_{16}$ & 6,8-Bisdehydro-4,5:10.11-dibenzopentatridecafulval-ene \\
\hline 16 & 40.04 & 280 & 3.05 & $\mathrm{C}_{18} \mathrm{H}_{32} \mathrm{O}_{2}$ & 9,12-Octadecadienoic acid (Z,Z) (Linoleic acid) \\
\hline 17 & 40.96 & 354 & 0.79 & $\mathrm{C}_{25} \mathrm{H}_{26} \mathrm{~N}_{2}$ & 8,9,10,11-Tetrahydro-8,8,11,11,15-pentamethylanchraceno [1,2-c]1,8-naphthridin \\
\hline 18 & 42.28 & 215 & 0.20 & $\mathrm{C}_{12} \mathrm{H}_{25} \mathrm{NO}_{2}$ & Octanoic acid, 2-dimethylaminoethyl ester \\
\hline 19 & 42.57 & 592 & 0.16 & $\mathrm{C}_{38} \mathrm{H}_{76} \mathrm{~N}_{2} \mathrm{O}_{2}$ & Octadecanamide, N,N'-1,2-ethanediylbis- \\
\hline 20 & 42.63 & 320 & 0.18 & $\mathrm{C}_{21} \mathrm{H}_{36} \mathrm{O}_{2}$ & 11,14,17-Eicosatrienoic acid, methyl ester (Methyl 11,14,17-icosatrienoate) \\
\hline 21 & 43.01 & 392 & 0.11 & $\mathrm{C}_{24} \mathrm{H}_{40} \mathrm{O}_{4}$ & Cholan-24-oic acid, 3,12-dihydroxy-, (3à,5á,12à) (Deoxycholic acid) \\
\hline 22 & 44.65 & 354 & 0.21 & $\mathrm{C}_{21} \mathrm{H}_{38} \mathrm{O}_{4}$ & $\begin{array}{l}\text { 9,12-Octadecadienoicacid } \quad \text { (Z,Z)-,2-hydroxy-1- (hydroxymethyl)ethyl ester } \\
\text { (Linolein, 2-mono-) }\end{array}$ \\
\hline 23 & 45.08 & 131 & 0.82 & $\mathrm{C}_{6} \mathrm{H}_{13} \mathrm{NO}_{2}$ & Acetic acid, 2- (dimethylamino)ethyl ester \\
\hline 24 & 46.36 & 540 & 1.58 & $\mathrm{C}_{30} \mathrm{H}_{36} \mathrm{O}_{9}$ & 8'-O-Ethyl-á-alectoronic acid \\
\hline 25 & 47.48 & 314 & 0.24 & $\mathrm{C}_{21} \mathrm{H}_{30} \mathrm{O}_{2}$ & Delta.-1 (2)- tetrahydrocannabinol \\
\hline 26 & 49.22 & 378 & 0.21 & $\mathrm{C}_{27} \mathrm{H}_{54}$ & Cyclohexane,1,3,5-trimethyl-2-octadecyl- \\
\hline 27 & 50.31 & 410 & 1.20 & $\mathrm{C}_{30} \mathrm{H}_{50}$ & Squalene \\
\hline 28 & 50.78 & 363 & 0.16 & $\mathrm{C}_{21} \mathrm{H}_{17} \mathrm{NO}_{3} \mathrm{~S}$ & 2-[ (2'-Acetylphenyl)amino]-5-[ (4"-methylphenyl)thio]-benzo[b]- $(1,4)$-quinone \\
\hline 29 & 52.81 & 376 & 0.62 & $\mathrm{C}_{24} \mathrm{H}_{24} \mathrm{~S}_{2}$ & Bis (6-methylthieno[3',2, 8,9]bicyclo[4.3.0]non- 1 (2)-en-3-ylidene) \\
\hline
\end{tabular}


Table 2. GC/MS analysis of n-hexane extract of Hericium erinaceus

\begin{tabular}{|c|c|c|c|c|c|}
\hline $\begin{array}{l}\text { Peak } \\
\text { No. }\end{array}$ & $\begin{array}{l}\text { Rt } \\
(\text { min.) }\end{array}$ & MW & $\begin{array}{l}\text { Area } \\
\%\end{array}$ & $\begin{array}{l}\text { Molecular } \\
\text { formula }\end{array}$ & Probabilities of the detected compounds \\
\hline 1 & 17.69 & 100 & 3.42 & $\mathrm{C}_{6} \mathrm{H}_{12} \mathrm{O}$ & Butanal, 2-ethyl- \\
\hline 2 & 20.58 & 168 & 4.39 & $\mathrm{C}_{11} \mathrm{H}_{20} \mathrm{O}$ & 1,7-Nonadien-4-ol, 4,8-dimethyl- \\
\hline 3 & 23.20 & 172 & 0.72 & $\mathrm{C}_{10} \mathrm{H}_{20} \mathrm{O}_{2}$ & Octanal, 7-hydroxy-3,7-dimethyl- \\
\hline 4 & 23.81 & 378 & 0.73 & $\mathrm{C}_{27} \mathrm{H}_{54}$ & Cyclohexane, 1,3,5-trimethyl-2-octadecyl- \\
\hline 5 & 28.33 & 186 & 0.80 & $\mathrm{C}_{11} \mathrm{H}_{22} \mathrm{O}_{2}$ & 1-Hydroxyundecan-10-one \\
\hline 6 & 28.66 & 126 & 0.50 & $\mathrm{C}_{9} \mathrm{H}_{18}$ & 3-Heptene, 2,6-dimethyl- \\
\hline 7 & 29.21 & 115 & 1.95 & $\mathrm{C}_{5} \mathrm{H}_{9} \mathrm{NO}_{2}$ & 3-Methyl-3-nitrobut-1-ene \\
\hline 8 & 29.44 & 115 & 0.71 & $\mathrm{C}_{5} \mathrm{H}_{9} \mathrm{NO}_{2}$ & 1-Pentene, 5-nitro- \\
\hline 9 & 29.96 & 112 & 7.02 & $\mathrm{C}_{7} \mathrm{H}_{12} \mathrm{O}$ & Allyl methallyl ether \\
\hline 10 & 31.38 & 744 & 0.42 & $\mathrm{C}_{16} \mathrm{H}_{8} \mathrm{Br}_{6} \mathrm{Cl}_{2}$ & (Z) and (E) à,á-Dibromo-3,3'-dichloro- 4,4'-bis (dibromomethyl) stilbene \\
\hline 11 & 31.82 & 138 & 14.23 & $\mathrm{C}_{10} \mathrm{H}_{18}$ & 1,6-Octadiene, 3,7-dimethyl- \\
\hline 12 & 33.46 & 662 & 0.54 & $\mathrm{C}_{39} \mathrm{H}_{42} \mathrm{~N}_{4} \mathrm{O}_{6}$ & $\begin{array}{l}\text { 34,38-Dioxo-33,35,36,37-tetramethoxy-31- methyl-3,7,23,27-tetraaza heptacyclo } \\
\text { (27.3.1.1.1.11.1)octaconta-1 (33),8-(35),9,11,13 (36),14,16,18 (37),19,21,29,31-dodecaene }\end{array}$ \\
\hline 13 & 33.70 & 144 & 1.08 & $\mathrm{C}_{8} \mathrm{H}_{16} \mathrm{O}_{2}$ & Heptanoic acid, methyl ester \\
\hline 14 & 34.44 & 140 & 0.42 & $\mathrm{C}_{9} \mathrm{H}_{16} \mathrm{O}$ & trans,-2,6-Nonadien-1-ol \\
\hline 15 & 35.00 & 186 & 0.72 & $\mathrm{C}_{11} \mathrm{H}_{22} \mathrm{O}_{2}$ & Nonanoic acid, ethyl ester \\
\hline 16 & 36.95 & 138 & 3.26 & $\mathrm{C}_{10} \mathrm{H}_{18}$ & Bicyclo [7.1.0]decane \\
\hline 17 & 37.07 & 140 & 1.78 & $\mathrm{C}_{10} \mathrm{H}_{20}$ & 1-Octene, 3,7-dimethyl- \\
\hline 18 & 37.18 & 131 & 0.46 & $\mathrm{C}_{6} \mathrm{H}_{13} \mathrm{NO}_{2}$ & Hexane, 1-nitro \\
\hline 19 & 38.16 & 138 & 4.51 & $\mathrm{C}_{10} \mathrm{H}_{18}$ & 1-Decyne \\
\hline 20 & 38.25 & 142 & 1.46 & $\mathrm{C}_{9} \mathrm{H}_{18} \mathrm{O}$ & 2-Nonen-1-ol, (E)- \\
\hline 21 & 38.36 & 126 & 1.14 & $\mathrm{C}_{9} \mathrm{H}_{18}$ & 1-Nonene \\
\hline 22 & 38.51 & 191 & 0.61 & $\mathrm{C}_{9} \mathrm{H}_{9} \mathrm{~N}_{3} \mathrm{O}_{2}$ & 1H-1,2,3-Triazole-4-carboxylic acid \\
\hline 23 & 38.67 & 117 & 1.54 & $\mathrm{C}_{5} \mathrm{H}_{11} \mathrm{NO}_{2}$ & Pentane, 1-nitro- \\
\hline 24 & 38.82 & 131 & 0.94 & $\mathrm{C}_{6} \mathrm{H}_{13} \mathrm{NO}_{2}$ & Hexane, 1-nitro- \\
\hline 25 & 41.92 & 184 & 0.44 & $\mathrm{C}_{12} \mathrm{H}_{24} \mathrm{O}$ & Z-2-Dodeceno \\
\hline 26 & 44.79 & 73 & 0.53 & $\mathrm{C}_{4} \mathrm{H}_{11} \mathrm{~N}$ & Isobutylamine \\
\hline 27 & 46.75 & 118 & 0.47 & $\mathrm{C}_{6} \mathrm{H}_{14} \mathrm{O}_{2}$ & Hydroperoxide, hexyl \\
\hline 28 & 48.32 & 112 & 0.75 & $\mathrm{C}_{8} \mathrm{H}_{16}$ & 1-Hexene, 3,3-dimethyl- \\
\hline 29 & 49.15 & 117 & 18.27 & $\mathrm{C}_{5} \mathrm{H}_{11} \mathrm{NO}_{2}$ & Pentane, 1-nitro- \\
\hline 30 & 52.06 & 118 & 4.02 & $\mathrm{C}_{6} \mathrm{H}_{14} \mathrm{O}_{2}$ & Hydroperoxide, 1-methylpentyl \\
\hline 31 & 53.39 & 102 & 7.47 & $\mathrm{C}_{6} \mathrm{H}_{14} \mathrm{O}$ & 3-Methyl-3-pentanol \\
\hline 32 & 56.31 & 118 & 4.37 & $\mathrm{C}_{6} \mathrm{H}_{14} \mathrm{O}_{2}$ & Hydroperoxide, 1-methylpentyl \\
\hline 33 & 56.52 & 119 & 0.44 & $\mathrm{C}_{4} \mathrm{H}_{9} \mathrm{NO}_{3}$ & 1-Butanol, 2-nitro \\
\hline
\end{tabular}

Note: $\mathrm{R}_{\mathrm{t}}$ : Retention time MW = Molecular weight

Table 3. GC/MS analysis of n-hexane extract of Dictyophora indusiata

\begin{tabular}{llllll}
\hline $\begin{array}{l}\text { Peak } \\
\text { No. }\end{array}$ & $\begin{array}{l}\text { Rt } \\
\text { min.) }\end{array}$ & $\mathbf{M W}$ & $\begin{array}{l}\text { Area } \\
\text { \% }\end{array}$ & $\begin{array}{l}\text { Molecular } \\
\text { formula }\end{array}$ & Probabilities of the detected compounds \\
\hline 1 & 11.82 & 100 & 0.07 & $\mathrm{C}_{6} \mathrm{H}_{12} \mathrm{O}$ & Butanal, 2-ethyl- \\
2 & 21.16 & 102 & 0.04 & $\mathrm{C}_{6} \mathrm{H}_{14} \mathrm{O}$ & 1-Pentanol, 2-methyl \\
3 & 31.60 & 116 & 0.04 & $\mathrm{C}_{6} \mathrm{H}_{12} \mathrm{O}_{2}$ & Hexanoic acid \\
4 & 33.68 & 130 & 0.11 & $\mathrm{C}_{7} \mathrm{H}_{14} \mathrm{O}_{2}$ & Hexanoic acid, methyl ester \\
5 & 34.99 & 186 & 0.05 & $\mathrm{C}_{11} \mathrm{H}_{22} \mathrm{O}_{2}$ & Nonanoic acid, ethyl ester \\
6 & 35.39 & 284 & 0.22 & $\mathrm{C}_{18} \mathrm{H}_{36} \mathrm{O}_{2}$ & Valeric acid, 4-tridecyl ester \\
7 & 36.96 & 117 & 10.47 & $\mathrm{C}_{5} \mathrm{H}_{11} \mathrm{NO}_{2}$ & Pentane, 1-nitro \\
8 & 37.06 & 131 & 4.63 & $\mathrm{C}_{6} \mathrm{H}_{13} \mathrm{NO}_{2}$ & Hexane, 1-nitro- \\
9 & 37.76 & 83 & 82.85 & $\mathrm{C}_{5} \mathrm{H}_{9} \mathrm{~N}$ & Butane, 1-isocyano- \\
10 & 37.97 & 117 & 0.19 & $\mathrm{C}_{5} \mathrm{H}_{11} \mathrm{NO}_{2}$ & Amyl nitrite \\
11 & 38.35 & 102 & 0.15 & $\mathrm{C}_{6} \mathrm{H}_{14} \mathrm{O}$ & Hexyl alcohol \\
12 & 39.87 & 102 & 0.08 & $\mathrm{C}_{6} \mathrm{H}_{14} \mathrm{O}$ & 3-Methyl-3-pentanol \\
13 & 40.78 & 160 & 0.04 & $\mathrm{C}_{8} \mathrm{H}_{16} \mathrm{O}_{3}$ & 5-Hexyl-1,2,4-trioxalane \\
14 & 43.13 & 86 & 0.05 & $\mathrm{C}_{5} \mathrm{H}_{10} \mathrm{O}$ & Cyclobutane, methoxy- \\
15 & 43.19 & 115 & 0.05 & $\mathrm{C}_{6} \mathrm{H}_{13} \mathrm{NO}_{1}$ & 2-Pentanone, 4-amino-4-methyl- \\
16 & 43.46 & 209 & 0.05 & $\mathrm{C}_{13} \mathrm{H}_{23} \mathrm{NO}_{2}$ & (Z)-6- (N,N-Diallylamino)-2-methyl-4-hexen-3-ol \\
17 & 46.97 & 142 & 0.05 & $\mathrm{C}_{5} \mathrm{H}_{6} \mathrm{~N}_{2} \mathrm{O}_{3}$ & 3,5-DIMETHYL-4-OXO-4H-PYRAZOLE1,2-DIOXIDE \\
18 & 47.07 & 671 & 0.05 & $\mathrm{C}_{29} \mathrm{H}_{39} \mathrm{Br}_{2} \mathrm{NO}_{7}$ & 1-Acetyl-4,4-bis[4- (3-bromopropoxy)-3,5-dimethoxyphenyl]piperidine \\
19 & 47.51 & 694 & 0.06 & $\mathrm{C}_{48} \mathrm{H}_{62} \mathrm{~N}_{4}$ & 2,7,12,17-Tetraethyl-3,5:8,10:13,15:18,20-tetrakis (2,2-dimethylpropano) porphyrin \\
20 & 49.14 & 114 & 0.05 & $\mathrm{C}_{8} \mathrm{H}_{16} \mathrm{O}_{2}$ & 5-Methyl-2-methylenehexane-1,4-diol \\
21 & 52.47 & 119 & 0.45 & $\mathrm{C}_{4} \mathrm{H}_{9} \mathrm{NO}_{3}$ & 1-Butanol, 2-nitro- \\
22 & 53.38 & 130 & 0.14 & $\mathrm{C}_{8} \mathrm{H}_{18} \mathrm{O}$ & 4-Methyl-3-heptanol \\
\hline
\end{tabular}




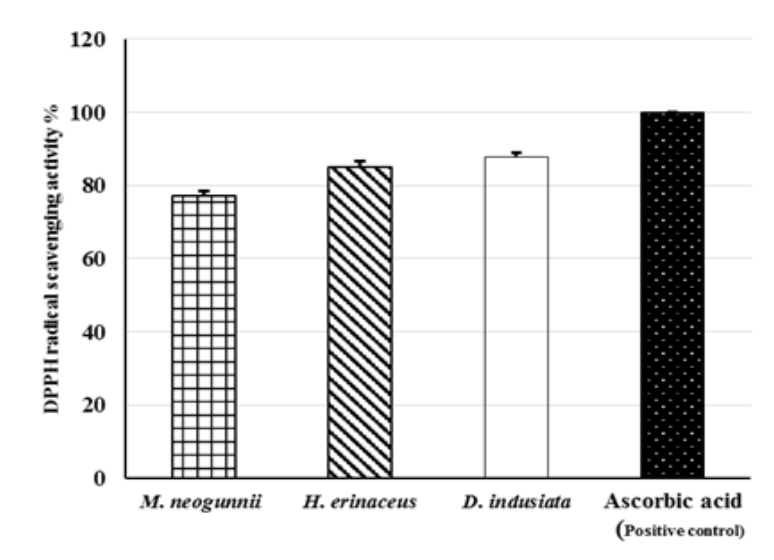

Figure 5. DPPH radical scavenging activity of n-hexane extracts of $H$. erinaceus, $M$. neogunnii, and $D$. indusiata at concentration $80 \mathrm{mg} / \mathrm{mL}$. Ascorbic acid at concentration $0.1 \%$ was used as positive control. Each value represents the mean of three replicates (Mean $\pm \mathrm{SD}$ )

\section{In vitro hypocholesterolemic activities of n-hexane mushrooms extracts.}

The effect of $D$. indusiata $H$. erinaceus, and $M$. neogunnii $\mathrm{n}$-hexane extracts on cholesterol concentration was evaluated using different concentrations of extracts and under incubation periods that reached 4 days at room temperature. The results shown in Table 4 revealed that the cholesterol reduction activities (CRA \%) of the three extracts is depending on the extract concentration as well as the incubation period. All extracts exerted potent in vitro cholesterol concentration lowering capabilities. Results ranged from $72.9 \pm 0.9 \%$ to $100 \pm 0 \%$ in case of $M$. neogunnii n-hexane extract, from $78.6 \pm 1.1 \%$ to $100 \pm 0 \%$ by using $H$. erinaceus $\mathrm{n}$-hexane extract, and from $77.9 \pm 1.1 \%$ to $100 \pm 0 \%$ when $D$. indusiata $\mathrm{n}$-hexane extract was used.

By finishing the first 24h, both extracts of $M$. neogunnii and D. indusiata at concentration of $32 \mathrm{mg} / \mathrm{mL}$ caused a higher reduction in cholesterol concentration $(89.3 \pm 1.3 \%$; and $89.3 \pm 1.7 \%$, respectively), in comparison with $H$. erinaceus extract which exhibited $88.6 \pm 1.4 \%$. Order of extracts potency using the same concentration $(32 \mathrm{mg} / \mathrm{mL})$ after 48 h were totally different, $H$. erinaceus extract came first recording $92.1 \pm 1.6 \%$, then $M$. neogunnii extract $(91.1 \pm 1.1 \%)$ followed by $D$. indusiata extract $(90.5 \pm 1.5 \%)$. By the third day, H. erinaceus extract continued its superiority over other extracts by recording a cholesterolreducing activity of $95.7 \pm 1.2 \%$, in comparison with $M$. neogunnii and $D$. indusiata extracts $(92.9 \pm 1.3 \%$, and $91.4 \pm 1.4 \%$ ) respectively. A promising total reduction in cholesterol concentration was achieved by the three extracts by the end of $96 \mathrm{~h}$.

\section{Discussion}

Many serious diseases such as diabetes, neurodegenerative, and cardiovascular diseases are developed pathologically by some free radicals. Generally, free radicals are generated as natural by-products from normal cellular metabolism of oxygen. Additionally, they

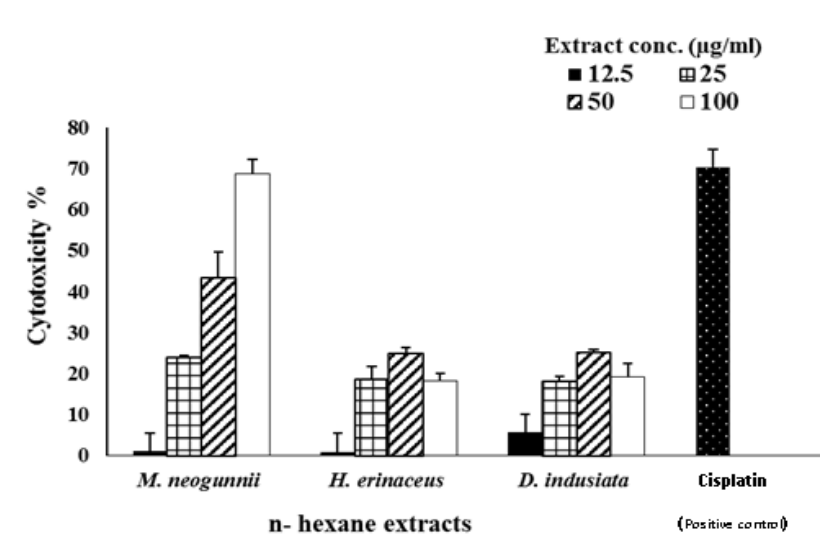

Figure 6. Cytotoxicity \% of $M$. neogunnii, H. erinaceus, and $D$. indusiata $\mathrm{n}$-hexane extract against HCT116 cell line monolayers. Cisplatin at concentration $50 \mu \mathrm{M}$ was used as positive control. Each value represents the mean of three independent experiments (Mean \pm SD)

can be induced by many factors like smoking, diet, lifestyle, stress, UV light, and ionizing radiation (Katerji et al. 2019). Free radicals are responsible for damaging proteins, membranes, and nucleic acids through several biological processes which result in many diseases (Bhattacharyya et al. 2014; Jiang et al. 2016). Moreover, there is a well-established relation between free radicals and cancer diseases (Sammar et al. 2019) which are major health challenges that threaten lives and rise annual mortalities worldwide. Hence, there is a serious need for screening for potent sources rich in antioxidants, which act as defense systems that regulate the levels of these free radicals. On the other hand, high cholesterol level is associated with an elevated risk of cardiovascular diseases (Elkhateeb et al. 2019b). Therefore, finding hypocholesterolemic potent sources are also of great need. Obviously, prevention and treating of such lethal diseases using natural edible sources would be of great significance to human health.

In the present study, it was aimed to compare the biological potencies of the mushrooms Hericium erinaceus, Metacordyceps neogunnii, and Dictyophora indusiata, through evaluating the in vitro antioxidant, anti-colon cancer and hypocholesterolemic properties of their nhexane extracts. These three mushrooms are edible mushrooms that are consumed for their beneficial nutritional characteristics.

Antioxidant properties of $n$-hexane extracts were investigated by testing their DPPH radical scavenging activities at $80 \mathrm{mg} / \mathrm{mL}$ concentration. All extracts showed promising antioxidant activities, which can be attributed to the richness of these extracts in fatty acid methyl esters which are known for exhibiting antioxidant activities (Pinto et al. 2017). Many studies had mentioned the antioxidant activities of the extracts of $H$. erinaceus (Charumathy et al. 2017; Lew et al. 2019); and D. indusiata (Oyetayo et al. 2009; Zhang et al. 2016). 
Table 4. In vitro cholesterol-reducing activity (CRA \%) of the n-hexane extracts of Metacordyceps neogunnii, Hericium erinaceus, and Dictyophora indusiata

\begin{tabular}{|c|c|c|c|c|c|c|c|c|c|c|c|c|}
\hline \multirow{2}{*}{$\begin{array}{c}\text { Extract } \\
\text { conc. } \\
(\mathrm{mg} / \mathrm{mL})\end{array}$} & \multicolumn{4}{|c|}{ M. neogunnii extract } & \multicolumn{4}{|c|}{$\begin{array}{c}\text { CRA\% } \\
\text { H. erinaceus extract } \\
\text { Incubation time (h) }\end{array}$} & \multicolumn{4}{|c|}{ D. indusiata extract } \\
\hline & 24 & 48 & 72 & 96 & 24 & 48 & 72 & 96 & 24 & 48 & 72 & 96 \\
\hline 4 & $72.9 \pm 0.9$ & $74.5 \pm 1.7$ & $77.1 \pm 1.2$ & $89.3 \pm 1.3$ & $78.6 \pm 1.1$ & $79.0 \pm 0.9$ & $79.3 \pm 0.8$ & $89.3 \pm 1.9$ & $77.9 \pm 1.1$ & $79.1 \pm 0.8$ & $80.0 \pm 0.4$ & $89.3 \pm 0.7$ \\
\hline 8 & $78.6 \pm 1.8$ & $79 \pm 1.3$ & $80.0 \pm 1.2$ & $95.7 \pm 1.8$ & $80.7 \pm 1.3$ & $81.0 \pm 1.0$ & $81.4 \pm 0.6$ & $93.6 \pm 1.1$ & $79.3 \pm 0.8$ & $80.4 \pm 1.4$ & $81.4 \pm 1.4$ & $91.4 \pm 0.6$ \\
\hline 16 & $82.1 \pm 1.6$ & $83.1 \pm 1.8$ & $83.6 \pm 2.1$ & $96.4 \pm 0.6$ & $83.6 \pm 1.4$ & $84.3 \pm 0.9$ & $85.0 \pm 0.8$ & $95.0 \pm 0.8$ & $85.0 \pm 0.8$ & $86.6 \pm 1.1$ & $87.9 \pm 1.4$ & $95.7 \pm 1.1$ \\
\hline 24 & $85.7 \pm 0.9$ & $86 \pm 0.7$ & $86.4 \pm 1.5$ & $99.3 \pm 0.3$ & $84.3 \pm 1.3$ & $85.7 \pm 1.3$ & $87.1 \pm 0.9$ & $95.7 \pm 1.2$ & $87.9 \pm 1.2$ & $89.3 \pm 1.3$ & $90.7 \pm 1.3$ & $98.6 \pm 0.6$ \\
\hline 32 & $89.3 \pm 1.3$ & $91.1 \pm 1.1$ & $92.9 \pm 1.3$ & $100.0 \pm 0$ & $88.6 \pm 1.4$ & $92.1 \pm 1.6$ & $95.7 \pm 1.2$ & $100.0 \pm 0$ & $89.3 \pm 1.7$ & $90.5 \pm 1.5$ & $91.4 \pm 1.4$ & $100.0 \pm 0$ \\
\hline
\end{tabular}

Each value represents the mean of three replicates (mean \pm SD) 
On the other hand, evaluating the anti-colon cancer activities of the extracts revealed potency of $M$. neogunnii extract at concentration of $100 \mu \mathrm{g} / \mathrm{mL}$ in comparison with other extracts at the same concentrations. Several in vivo and in vitro studies have described the anticancer activities of $D$. indusiata extracts against group of human cancer cell lines (Liao et al. 2015; Han et al. 2017), also the anticancer activities of extract is well known (Kim et al. 2013; Li et al. 2014; Am, 2017). The in vitro hypocholesterolemic studies which were conducted on the three extracts showed their promising capabilities in cholesterol-reducing. All extracts succeeded in lowering cholesterol concentrations in $100 \%$ after incubation for 4 days at room temperature. At the third day, M. neogunnii n-hexane was the fastest in reaching the highest reducing ability by recording $99.3 \pm 0.3 \%$. It was previously reported that many mediumchain fatty acids as those detected in the extracts are capable of reducing cholesterol levels (Liu et al. 2017). It should be noted that studies on antioxidant, anti- colon cancer, and hypocholesterolemic activities of M. neogunnii extract are relatively rare, and this is the first study that describes its antioxidant activities.

Chemical analyses on the different extracts were performed in order to describe the metabolic profiles of the extracts, which may help in understanding the secondary metabolites originated from those mushrooms and their role in the observed biological activities. Medium-chain fatty acid such as caproic acid, which was detected in the nhexane extract of $D$. indusiata has significant anticancer and anti-proliferation activities against human colorectal, skin and breast cancer cells and could potentially be used to prevent and/or treat these cancers (Narayanan et al. 2015). Compound such as Cyclohexane, 1,3,5-trimethyl-2octadecyl- which was detected in $H$. erinaceus extract has anticancer activity (Viswanathan, 2014). In general, results in our study suggested an overall superiority of $M$. neogunnii extract. GC-Ms analysis of $M$. neogunnii nhexane extract revealed the presence of 13 ester compounds out of the 29 detected compounds which came in accordance with results described by She et al (2019). Promising biological activities exerted by $M$. neogunnii extract can be explained by the presence of many bioactive compounds representing major peak areas detected by GCMs analysis (Table 1). Palmitic acid methyl ester (representing $11.75 \%$ of total peak area) is known for its antioxidant, hypocholesterolemic activities among many other activities (Mujeeb et al. 2014). On the other hand, 9 Octadecenoic acid (Z), methyl ester which was detected as major peak $(50.74 \%)$ in Metacordyceps extract has strong anticancer and antioxidant activities (Yeong et al. 1989; Asghar et al. 2011; Belakhdar et al. 2015). Moreover, 9 Octadecenoic acids (Z), methyl ester exhibits hypocholesterolemic effect (Krishnamoorthy and Subramaniam, 2014). Another ester (9, 12- octadecadienoic acid (Z, Z)-, methyl ester) was detected in this extract (representing $15.94 \%$ of total peak area) is known for its antioxidant and anticancer properties (Kumar et al. 2010; Wei et al. 2011). Finally, 9,12 Octadecadienoic acid (Z,Z) (Linoleic acid ( which was detected in $3.05 \%$ peak area is also characterized by having promising antioxidant activities (Peyrat-Maillard et al. 2003; Rahman et al. 2014). Furthermore, Dommels et al (2003) described the effect of high doses of linoleic acid on inhibiting colon cancer cell line Caco-2 proliferation. In another study, a protective effect against cancer development was accomplished by high intake of fatty acids such as linoleic acid (Horrobin and Ziboh, 1997). A synergism between those different compounds can explain the recorded promising biological activities of those mushrooms extracts.

In conclusion, the present study provides encouraging data for using extracts of $H$. erinaceus, $M$. neogunnii, and D. indusiata in the pharmaceutical industry, and for disease prevention. An advantage that facilitates doing that is the fact that all tested mushrooms in this study are already edible mushrooms which also support their use as functional foods. Additionally, the broad therapeutic values of these mushrooms reserve promising future for them in modern alternative medicine. Further studies are encouraged to understand the mode of action behind the properties of their bioactive compounds, and to explain possible synergism between such compounds which resulted in these promising results. Furthermore, finding a natural edible source having multiple biological activities against different life-threatening diseases as colon-cancer, cardiovascular diseases caused by high cholesterol levels, as well as exhibiting promising antioxidant properties is this era target in order to win the human battle against such life-threatening diseases.

\section{REFERENCES}

Adams RP. 1995. Identification of Essential Oil Components By Gas Chromatography/ Mass Spectroscopy. 4th ed. Allured Publishing Corporation, Carol Stream, IL.

AM Y. 2017. Anticancer potential of Hericium erinaceus extracts against particular human cancer cell lines. Microb Biosys 2 (1): 9-20.

Asghar SF, Choudahry MI. 2011. Gas chromatography-mass spectrometry (GC-MS) analysis of petroleum ether extract (oil) and bio-assays of crude extract of Iris germanica. Intl J Genet Mol Biol 3 (7): 95-100.

Bashir R, Palombo E, Mahon P. 2017. Bioactive profile of Australian native Cordyceps gunnii $5^{\text {th }}$ International Conference and Exhibition on Pharmacognosy, Phytochemistry \& Natural Products, Melbourne.

Belakhdar G, Benjouad A, Abdennebi EH. 2015. Determination of some bioactive chemical constituents from Thesium humile Vahl. J Mater Environ Sci. 6 (10): 2778-2783.

Bhattacharyya A, Chattopadhyay R, Mitra S, Crowe SE. 2014. Oxidative stress: an essential factor in the pathogenesis of gastrointestinal mucosal diseases. Physiol Rev 94 (2): 329-354.

Chang ST, Miles PG. 1989. Edible Mushrooms and their Cultivations. Boca Raton, FL: CRC Press Inc., Boca Raton, FL.

Charumathy M, Sudha G, Packialakshmi B. 2017 In vitro antioxidant activity analysis in Hericium erinaceus pers fruiting bodies Int $\mathrm{J}$ Pharm Technol 9: 30118-30128.

Dommels YE, Haring MM, Keestra NG, Alink GM, van Bladeren PJ, van Ommen B. 2003. The role of cyclooxygenase in $n-6$ and $n-3$ polyunsaturated fatty acid-mediated effects on cell proliferation, PGE 2 synthesis and cytotoxicity in human colorectal carcinoma cell lines. Carcinogenesis 24 (3): 385-392.

Elkhateeb WA, Daba GM, Sheir D, El-Dein AN, Fayad W, Elmahdy EM, Shaheen MN, Thomas PW, Wen TC. 2019c. GC-MS analysis and invitro hypocholesterolemic, anti-rotavirus, anti-human colon carcinoma activities of the crude extract of a Japanese mushroom Ganoderma spp. Egypt Pharm J 18: 102-110.

Elkhateeb WA, Daba GM, Thomas PW, Wen TC. 2019a. Medicinal mushrooms as a new source of natural therapeutic bioactive compounds. Egypt Pharm J 18 (2): 88-101. 
Elkhateeb WA, Elnahas MO, Thomas PW, Daba GM. 2019b. To heal or not to heal? Medicinal mushrooms wound healing capacities. ARC J Pharm Sci 5 (4): 28-35.

Elkhateeb WA, Elnahas MO, Thomas PW, Daba GM. 2020. Trametes versicolor and Dictyophora indusiata, champions of medicinal mushrooms. Open access J Pharm Res 4 (1): 1-7.

Golomb BA, Evans MA. 2008. Statin adverse effects. Am J Cardiovasc Drugs 8 (6): $373-418$

Gong M, An J, Lu HZ, Wu CF, Li YJ, Cheng JQ, et al. 2004. Effects of denaturation and amino acid modification on fluorescence spectrum and hemagglutinating activity of Hericium erinaceum lectin. Acta Biochim Biophys Sin. 36 (5): 343-50

Han S, Ma C, Hu M, Wang Y, Ma F, Tao N, Qin Z. 2017. A polysaccharide from Dictyophora indusiata inhibits the immunosuppressive function of cancer-associated fibroblasts. Cell Biochem Funct 35 (7): 414-419.

Horrobin DF, Ziboh VA. 1997. The importance of linoleic acid metabolites in cancer metastasis and in the synthesis and actions of 13-HODE. Adv. Exp. Med Biol. 433: 291-294.

Jeong SM, Choi S, Kim K, Kim SM, Lee G, Park SY, Kim YY, Son JS, Yun JM, Park SM. 2018. Effect of change in total cholesterol levels on cardiovascular disease among young adults. J Am Heart Assoc 7 (12): e008819. DOI: 10.1161/JAHA.118.008819.p.e008819.

Jiang S, Wang Y, Zhang X. 2016. Comparative studies on extracts from Hericium erinaceus by different polarity reagents to gain highe antioxidant activities. Exp Ther Med 12 (1): 513-517.

Katerji M, Filippova M, Duerksen-Hughes P. 2019. Approaches and Methods to Measure Oxidative Stress in Clinical Samples: Research Applications in the Cancer Field. Oxid Med Cell Longev, 2019.1-29.

Kim SP, Nam SH, Friedman M. 2013. Hericium erinaceus (Lion's Mane) mushroom extracts inhibit metastasis of cancer cells to the lung in CT-26 colon cancer-transplanted mice. J Agric Food Chem 61 (20): 4898-4904

Krishnamoorthy K, Subramaniam P. 2014. Phytochemical profiling of leaf, stem, and tuber parts of Solena amplexicaulis (Lam.) Gandh using GC-MS. Intl Sch Res Notices 2014:1-13.

Kumar PP, Kumaravel S, Lalitha C. 2010. Screening of antioxidant activity, total phenolics and GC-MS study of Vitex negundo. Afr J Biochem Res. 4 (7): 191-195

Lee B, Kim J, Kang YM, Lim J, Kim Y, Lee M, Min-Ho J, Chang-Bum A, Jae-Young Je. 2010. Antioxidant activity and $\gamma$-aminobutyric acid (GABA) content in sea tangle fermented by Lactobacillus brevis BJ20 isolated from traditional fermented foods. Food Chem. 122: 271-276.

Lew SY, Yow YY, Lim LW, Wong KH. 2019. Antioxidant-mediated protective role of Hericium erinaceus (Bull.: Fr.) Pers. against oxidative damage in fibroblasts from Friedreich's ataxia patient. Food Sci Technol 13: 1-9.

Li G, Yu K, Li F, Xu K, Li J, He S, Cao S, Tan G. 2014. Anticancer potential of Hericium erinaceus extracts against human gastrointestinal cancers. J Ethnopharmacol 153 (2): 521-530.

Li Y, Zhang G, Ng TB, Wang H. 2010. A novel lectin with antiproliferative and HIV-1 reverse transcriptase inhibitory activities from dried fruiting bodies of the monkey head mushroom Hericium erinaceum. BioMed Res Intl 2010: 716515. DOI: 10.1155/2010/716515.1-9.

Liang ZQ. 1985. Isolation and identification of the conidial stage of Cordyceps gunnii. Acta Mycologica Sinica 4 (3): 162-166.

Liao W, Lu Y, Fu J, Ning Z, Yang J, Ren J. 2015. Preparation and characterization of Dictyophora indusiata polysaccharide-zinc complex and its augmented antiproliferative activity on human cancer cells. J Agric Food Chem 63 (29): 6525-6534.

Liu Y, Zhang Y, Zhang X, Xu Q, Yang X, Xue C. 2017. Medium-chain fatty acids reduce serum cholesterol by regulating the metabolism of bile acid in C57BL/6J mice. Food Funct 8 (1): 291-298.

Mizuno T, Wasa T, Ito H, Suzuki C, Ukai N. 1992. Antitumor-active polysaccharides isolated from the fruiting body of Hericium erinaceum, an edible and medicinal mushroom called yamabushitake or houtou. Biosci Biotechnol Biochem 56 (2): 347-8.

Mujeeb F, Bajpai P, Pathak N. 2014. Phytochemical evaluation, antimicrobial activity, and determination of bioactive components from leaves of Aegle marmelos. BioMed Res Intl 2014:497606. DOI: 10.1155/2014/497606.2014: 1-11

Narayanan A, Baskaran S, Amalaradjou M, Venkitanarayanan K. 2015. Anticarcinogenic properties of medium-chain fatty acids on human colorectal, skin and breast cancer cells in vitro. Intl J Mol Sci 16 (3) 5014-5027.
Oyetayo VO, Dong CH, Yao YJ. 2009. Antioxidant and antimicrobial properties of aqueous extract from Dictyophora indusiata. Open Mycol J 3 (1): 20-26.

Pan J, Zhang Q, Zhang Y, Ouyang Z, Zheng Q, Zheng R. 2005. Oxidative stress in heroin administered mice and natural antioxidants protection. Life Sci. 27, 77 (2), 1: 83-93.

Peyrat-Maillard MN, Cuvelier ME, Berset C. 2003. Antioxidant activity of phenolic compounds in 2, 2'-azobis (2-amidinopropane) dihydrochloride (AAPH)-induced oxidation: Synergistic and antagonistic effects. J Amer Oil Chem Soc 80: 1007. DOI: 10.1007/s11746-003-0812-zJ Am Oil Chem Soc 80 (10): p.1007.

Pinto ME, Araujo SG, Morais MI, Sá NP, Lima CM, Rosa CA, Siqueira EP, Johann S, Lima LA. 2017. Antifungal and antioxidant activity of fatty acid methyl esters from vegetable oils. An Acad Bras Ciênc 89 (3): 1671-1681

Rahman MA, Abdullah N, Aminudin N. 2014. Inhibitory effect on in vitro LDL oxidation and HMG Co-A reductase activity of the liquid-liquid partitioned fractions of Hericium erinaceus (Bull.) Persoon (lion's mane mushroom). BioMed Res Int 2014: 1-9.

Sammar M, Abu-Farich B, Rayan I, Falah M, Rayan A. 2019. Correlation between cytotoxicity in cancer cells and free radical-scavenging activity: In vitro evaluation of 57 medicinal and edible plant extracts. Oncol Lett 18 (6): 6563-6571.

She X, Yao T, Ding L, Gan Z, Tang M, Han Y. 2019. Identification and Analysis of Secondary Metabolites from $\mathrm{CH}_{3} \mathrm{OH} / \mathrm{H}_{2} \mathrm{O}$ Extract of Metacordyceps neogunnii. E3S Web Conf 118: 01027. DOI: 10.1051/e3sconf/201911801027In E3S Web of Conferences (Vol. 118, p. 01027). EDP Sciences.

Sullivan, R., Smith, J.E., Rowan, N.J. 2006. Medicinal mushrooms and cancer therapy: translating a traditional practice into Western medicine. Perspect Biol Med 49 (2): 159-170.

Thompson PD, Panza G, Zaleski A, Taylor B. 2016. Statin-associated side effects. J Am Coll Cardiol 67: 2395-2410.

Thongbai B, Rapior S, Hyde KD, Wittstein K, Stadler M. 2015. Hericium erinaceus, an amazing medicinal mushroom. Mycol Prog 14 (10): 91-113.

Viswanathan SG. 2014. GC-MS Analysis of Phytocomponents phytocomponents in Spermacoce articularis L. f. leaf. Res Pharm 4 (4): 1-7.

Wang XY, Yin JY, Nie SP, Xie, M.Y. 2018. Isolation, purification and physicochemical properties of polysaccharide from fruiting body of Hericium erinaceus and its effect on colonic health of mice. Intl Biol Macromol 107: 1310-1319.

Wei LS, Wee W, Siong JY, Syamsumir DF. 2011. Characterization of anticancer, antimicrobial, antioxidant properties and chemical compositions of Peperomia pellucida leaf extract. Acta Medica Iranica 49 (10): 670-674

Wen TC, Xiao YP, Han YF, Huang SK, Zha LS, Hyde KD, Kang JC. 2017. Multigene phylogeny and morphology reveal that the Chinese medicinal mushroom Metacordyceps neogunnii sp. nov. Phytotaxa 302 (1): 27-39.

WHO. 2017. World Health Organization cardiovascular-diseases- (cvds) fact sheet, 17 May 2017. http://www.who.int/en/news-room/factsheets/detail/cardiovascular-diseases- (cvds).

WHO. 2018. World Health Organization fact sheet, 12 September 2018. https://www.who.int/news-room/fact-sheets/detail/cancer.

Xiao Z, Storms R, Tsang A. 2006. A quantitative starch-iodine method for measuring alpha-amylase and glucoamylase activities. Anal Biochem 351 (1): 146-148.

Yang CH, Kao YH, Huang KS, Wang CY, Lin LW. 2012. Cordyceps militaris and mycelial fermentation induced apoptosis and autophagy of human glioblastoma cells. Cell Death Dis 3:1-9.

Yeong LH, Nancy KG, Micheal WP. 1989. Newly recognized anticarcinogenic fatty acids: identification and quantification in natural and processed cheeses: J. Agric. Food Chem. 37: 75-81.

Yim MH, Shin JW, Son JY, Oh SM, Han SH, Cho JH, et al. 2007. Soluble components of Hericium erinaceum induce NK cell activation via production of interleukin-12 in mice splenocytes. Acta Pharmacol Sin. 28 (6): 901-907.

Zhang J, Shi R, Li H, Xiang Y, Xiao L, Hu M, Ma F, Ma CW, Huang Z. 2016. Antioxidant and neuroprotective effects of Dictyophora indusiata polysaccharide in Caenorhabditis elegans. $\mathrm{J}$ Ethnopharmacol 192: 413-422.

Zhang Z, Lv G, Pan H, Pandey A, He W, Fan L. 2012. Antioxidant and hepatoprotective potential of endo-polysaccharides from Hericium erinaceus grown on tofu whey. Intl J Biol Macromol. 51 (5): 11401146. 
BIODIVERSITAS

Volume 21, Number 6, June 2020

Pages: 2437-2445
ISSN: 1412-033X

E-ISSN: 2085-4722

DOI: 10.13057/biodiv/d210615 